\title{
Patterned Biofunctional Poly(Acrylic Acid) Brushes on
}

\section{Silicon Surfaces}

\author{
Rong Dong, ${ }^{1,2}$ Sitaraman Krishnan, ${ }^{2}$ Barbara A. Baird, ${ }^{1}$ Manfred Lindau, ${ }^{3}$ Christopher K. Ober ${ }^{2, *}$
}

Department of Chemistry and Chemical Biology, Department of Materials Science and Engineering, Department of Applied and Engineering Physics, Cornell University, Ithaca, NY 14853

TITLE RUNNING HEAD: Patterned Biofunctional Poly(Acrylic acid) Brushes on Silicon Surfaces

* To whom correspondence should be sent. E-mail: cober@ccmr.cornell.edu; Tel 607-255-8417; Fax 607-255-2365.

${ }^{1}$ Department of Chemistry and Chemical Biology

${ }^{2}$ Department of Materials Science and Engineering

${ }^{3}$ Department of Applied and Engineering Physics 
X-ray photoelectron spectroscopy (XPS) measurements were performed using a SSX-100 instrument (Surface

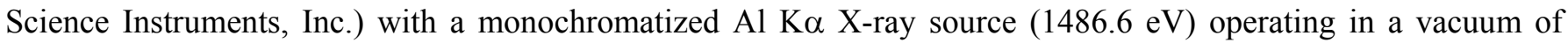
$1 \times 10^{-9}$ Torr. The spectra were acquired at an electron emission angle of $0^{\circ}$ (electron emission along the surface normal). Charge neutralization was carried out by injection of low energy electrons, and the $\mathrm{C}-\mathrm{C} 1 \mathrm{~s}$ peak was corrected to a binding energy of $285 \mathrm{eV}$. Deconvolution of the XPS spectra of the BSA-functionalized surfaces was performed using the procedure of Bomben and Dev, with an additional $-\mathrm{COOH}$ peak at $\sim 289 \mathrm{eV}$.
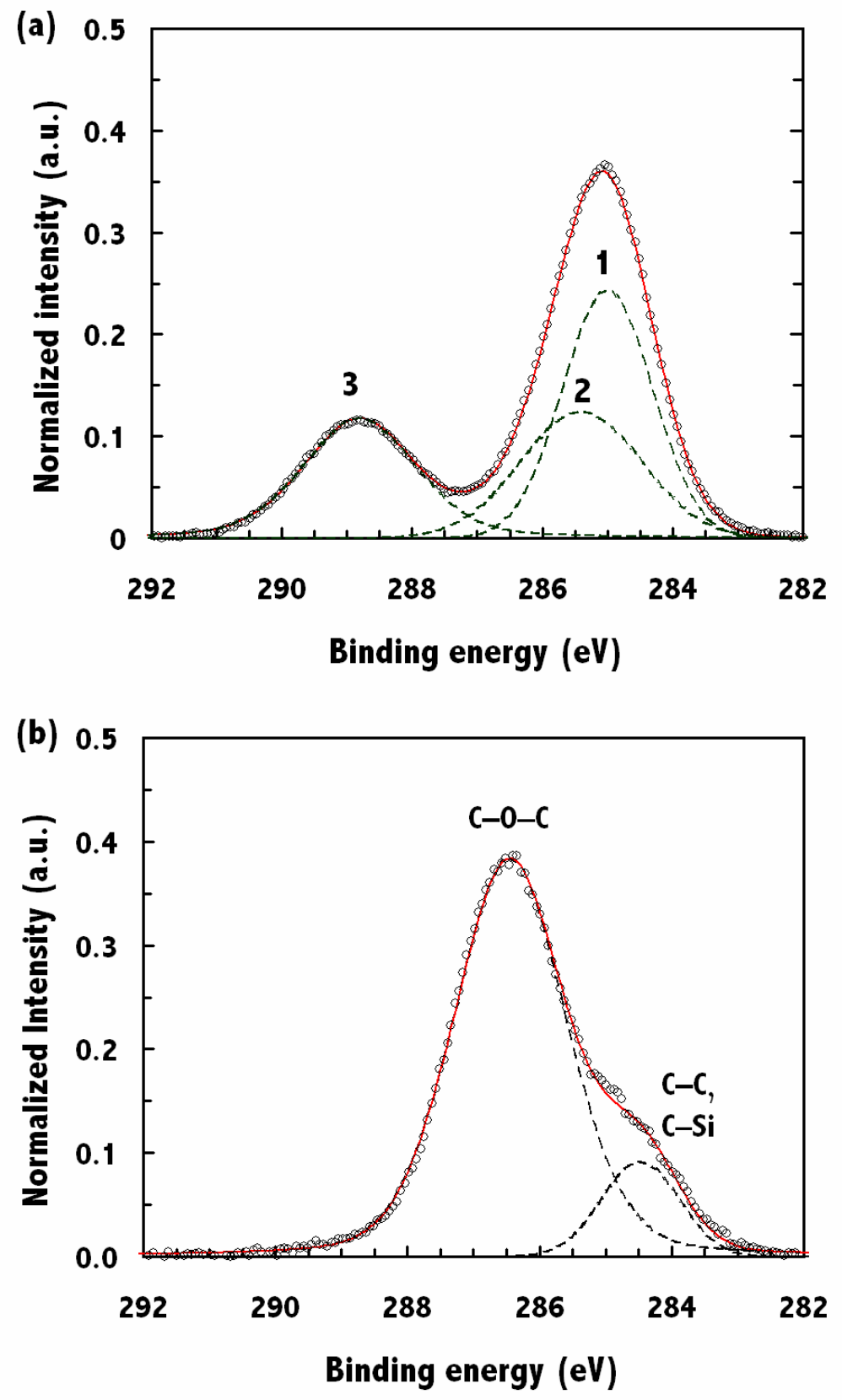

Figure 1. XPS spectra of (a) poly(acrylic acid) (PAA) brush, and (b) PEG SAM on silicon surfaces. Sub-peaks 1 and 2 in (a) arise from alkyl carbon atoms along the PAA backbone, while peak 3 is due to $-\underline{\mathrm{C}}(=\mathrm{O})-\mathrm{OH}$. Methoxy(polyethylenoxy)propyl]trichlorosilane, $\mathrm{CH}_{3} \mathrm{O}\left(\mathrm{CH}_{2} \mathrm{CH}_{2} \mathrm{O}\right)_{6-9}\left(\mathrm{CH}_{2}\right)_{3} \mathrm{SiCl}_{3}$, was used to prepare the PEG $\mathrm{SAM}$. The hydrocarbon peak in (b) is from the propyl spacer of this molecule. The relative area of the $\mathrm{C}-\mathrm{O}-\mathrm{C}$ ether peak is $87 \%$, which is in accord with the chemical structure of the silane. 

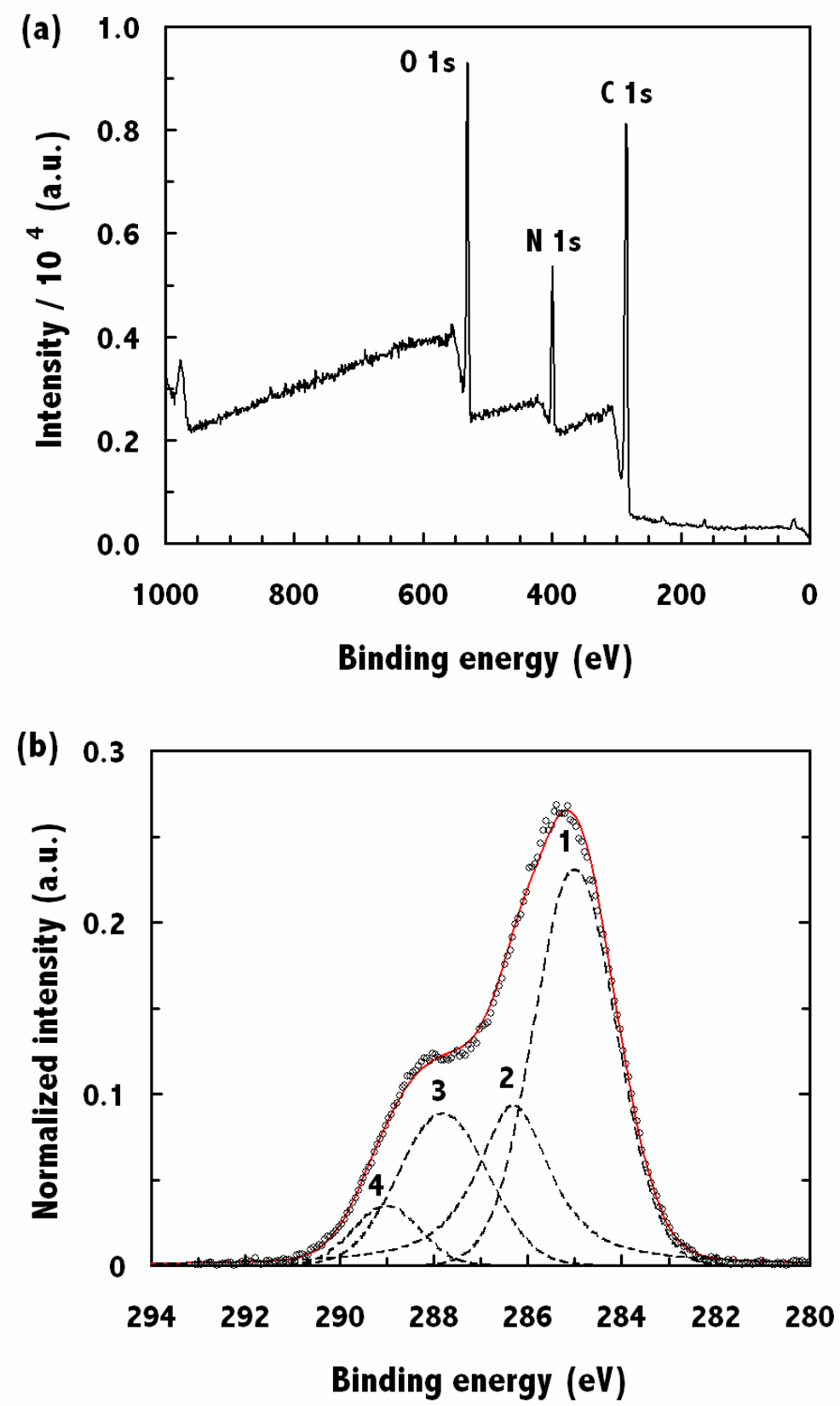

Figure 2. (a) XPS survey scan of covalently immobilized bovine serum albumin on PAA brush surface, and (b) the high resolution $\mathrm{C}$ 1s spectrum, resolved into component peaks. The peak assignments are: (1) $\underline{\mathrm{C}}-\mathrm{C}, \underline{\mathrm{C}}-\mathrm{H}$ (2) $\underline{\mathrm{C}}-\mathrm{N}$ and $\underline{\mathrm{C}}-\mathrm{O}$ (3) $\underline{\mathrm{C}}=\mathrm{O}$ (amide), and (4) $\underline{\mathrm{C}}=\mathrm{O}$ (carboxylic acid). The circles represent the experimental data points. The continuous line is the best-fit curve. The dashed curves are fitted sub-peaks. The relative area of the carboxylic acid carbon atoms (peak 5) is only about $4 \%$, indicating a dense immobilization of BSA within the top $3 \mathrm{~nm}$ of the brush surface. The high degree of protein immobilization is also evident in the relatively intense $\mathrm{N} 1 \mathrm{~s}$ peak in the survey scan.

\footnotetext{
${ }^{1}$ Bomben, K. D.; Dev, S. B. Investigation of poly(L-amino acids) by X-ray photoelectron spectroscopy. Anal. Chem. 1988, 60, 1393-1397.
} 